\title{
EXTENSION OF LEGENDER'S FORMULA TO THE DOUBLE FACTORIAL AND GENERALIZE IT TO THE MULTIFACTORIAL
}

\author{
HAMZAH ALTAAI
}

Research Scholar, Department of Mathematics, College of Science, University of Baghdad, Iraq

ABSTRACT
Legendre introduced the formulae of exponent of prime numbers forming factorial. Larry Tesler put a limit to the
Legendre formulae. In this paper, two theorems have been introduced. In the first theorem, a new formula has been
derived that describes the exponent of the prime numbers those factors in the double factorial (n!!), with its proof. In
second theorem, a new formula has been derived that describes the exponent of the prime numbers that factors in the
Multi factorial (n!q), with its proof. The formulae in the theorems are derived using an innovative methods based on
the Legendre and Larry Tesler principles.
KEYWORDS: Factorials, Double Factorial, Multi Factorial, Primes, Legendre's Formula, Larry Tesler, Floor
Function

Received: Jun 01, 2020; Accepted: Jun 21, 2020; Published: Aug 14, 2020; Paper Id.: IJMCARDEC20201

\section{INTRODUCTION}

The factorial of integer number such as (n) is written as (n!) the multiplication of (n) by all the numbers less than it down to (1). It is written as [1][2]:

$$
\begin{aligned}
& n !=1 \times 2 \times 3 \times 4 \times 5 \times \ldots \ldots \ldots \times n \\
& =\prod_{k=1}^{n} k
\end{aligned}
$$

Assume: $p_{1}, p_{2}, p_{3}, p_{4}, p_{5}, \ldots \ldots \ldots \ldots p_{m}$ as prime numbers between (1) and $(\mathrm{n})$, where $(\mathrm{m})$ is the number of primes, the factorial $(n$ !) can be written as:

$$
p_{1}^{z_{1}} \times p_{2}^{z_{2}} \times p_{3}^{Z_{3}} \times p_{4}^{Z_{4}} \times p_{5}^{Z_{5}} \times \ldots \ldots \ldots \ldots p_{m}^{z_{m}}
$$

Where all the numbers between (1) and (n) are essentially composed of the primes between (1) and (n) (see [3]).

Legendre's formula (Adriane Mary Legendre, French Scientist, 1752-1833 AD) describe the exponent of prime that factor in (n!). The formula is written as [4]:

$$
v_{p}(n !)=\sum_{k=1}^{\infty}\left\lfloor\frac{n}{p^{k}}\right\rfloor=\left\lfloor\frac{n}{p}\right\rfloor+\left\lfloor\frac{n}{p^{2}}\right\rfloor+\left\lfloor\frac{n}{p^{3}}\right\rfloor+\ldots
$$

Where:

$$
\begin{aligned}
& p \text { is prime number between }(\mathrm{n}) \text { and }(1) . \\
& v_{p}(n !) \text { is Exponent of prime number }(\mathrm{p}) \text { factor in }(\mathrm{n} !) \\
& \left.\left\lfloor\frac{n}{p^{k}}\right\rfloor \text { is floor function to } \frac{n}{p^{k}} \text { (see }[5]\right)
\end{aligned}
$$




\section{Proof:}

Since $n$ ! is the result of multiplication of all the integers from (1) to (n) so we can get at least one factor, from each multiple of (p) between (n) and (1) by dividing (n) by (p) and getting an additional prime element from each dividend of $\left(p^{2}\right)$ between $(n)$ and $(1)$ by dividing $(n)$ by $\left(p^{2}\right)$ and continue to get another additional factor of each multiple $\left(p^{3}\right)$ between (n) and (1) by the division of (n) by $\left(\mathrm{p}^{3}\right)$ and so on. By sum of the terms, we get the exponent of the prime (p) in (n!) [4].

In [6] Larry Tesler (1961) introduced a formula to limit the summation of Legendre formula by the value of

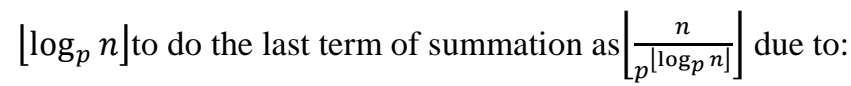

$$
\begin{aligned}
& \left\lfloor\log _{p} n\right\rfloor \leq \log _{p} n<\left\lfloor\log _{p} n\right\rfloor+1 \\
& \Rightarrow p^{\left.\mid \log _{p} n\right\rfloor} \leq n<p^{\left\lfloor\log _{p} n\right\rfloor+1} \\
& \therefore\left|\frac{n}{p^{\left.\mid \log _{p} n\right\rfloor}}\right| \geq 1 \\
& \text { And }\left|\frac{n}{p^{\left|\log _{p} n\right|+1}}\right|=0
\end{aligned}
$$

Therefore no need to add other terms such as $k>\left\lfloor\log _{p} n\right\rfloor$ because all should be zero

$$
\left\lfloor\frac{n}{p^{k}}\right\rfloor=0
$$

The Larry Tesler's formula was as in follow:

$$
v_{p}(n !)=\sum_{k=1}^{\left\lfloor\log _{p} n\right\rfloor}\left\lfloor\frac{n}{p^{k}}\right\rfloor
$$

In this paper, two theorems have been introduced; in the first theorem a new formula has been derived that describes the exponent of the prime numbers those factors in the double factorial (n!!), and its proof. In second theorem a new formula has been derived that describes the exponent of the prime numbers that factors in the Multifactorial $\left(n !^{q}\right)$, and its proof. The formulae in the theorems arederived in an innovative method based on the Legendre and Larry Tesler principles.

\section{EXTENSION OF LEGENDER'S FORMULA TO THE DOUBLE FACTORIAL}

\subsection{Definition of Double Factorial}

The mathematical expression (n!!) is a double factorial and it is the product of the integers between (n) and (1) that have the same odd or even properties of (n). It means, if (n) is odd, (n !!) is the product of odd numbers between (n) and (1), where it is by the same mannerif (n) is even. However (n!!) formula is written as follow [7][8]:

$n ! !=n(n-2)(n-4)(n-6) \ldots \ldots\left(n-2\left(\left\lceil\frac{n}{2}\right\rceil-1\right)\right)=\prod_{k=0}^{\left\lceil\frac{n}{2}\right\rceil-1}(n-2 k)$

Assume that the set of prime numbers between (1) and (n) are: $p_{1}, p_{2}, p_{3}, p_{4}, p_{5}, p_{6}, p_{7}, \ldots \ldots \ldots \ldots, p_{m}$

Where $m$ is the number of prime numbers.

Since the numbers between (1) and (n) are essentially formed by prime numbers between (1) and (n) (see [3]), then double factorial can be written as: 


$$
p_{1}^{z_{1}} \times p_{2}^{Z_{2}} \times p_{3}^{Z_{3}} \times p_{4}^{z_{4}} \times p_{5}^{z_{5}} \times p_{6}^{z_{6}} \times p_{7}^{z_{7}} \times \ldots \ldots \times p_{m}^{z_{m}}
$$

\subsection{ProposedTheorem}

Exponent of those prime numbers that are the factors in forming double factorial (n!!) is given by the following relationship:

$v_{p}(n ! !)=\sum_{k=1}^{\left\lfloor\log _{p} n\right\rfloor} \frac{\left\lfloor\frac{n}{p^{k}}\right\rfloor+\frac{(-1)^{n+1}+(-1)^{\left\lfloor\frac{n}{p^{k}}\right\rfloor}+n}{2}}{2}$

Where:

$\mathrm{p}$ is prime number between (1) and (n)

$v_{p}(n !)$ Is the exponent of $\mathrm{p}$ the factor in $(\mathrm{n} ! !)$

\section{Proof:}

Step 1: In general the positive multiples of prime numbers ( $\mathrm{p})$ are written as: $p, 2 p, 3 p, 4 p, 5 p, 6 p, 7 p, \ldots \ldots \ldots$

It is a multiplication operation of $\mathrm{p}$ by positive integers. So due to the alternating nature of integer numbers as odd even, odd even and so on. Then the positive multiples of prime numbers (p) are in the same context as odd even, odd even and so on too. The number $\mathrm{p}$ is prime as well as it is odd at all values (except number 2), anyway no effect on the rule. By dividing of $n$ by $p$, number of multiples of (p) between (1) and (n) is obtained. As first divided number is odd because it is assigned (p) and the last one between (1) and (n) is $\mathrm{p}\left\lfloor\frac{\mathrm{n}}{\mathrm{p}}\right\rfloor$ in addition to the alternating nature of the numbers, then:

- If the number of multiples of (p) between (1) and (n) is even, then the odd and even number of (p) multiples are equalized, no matter if (n) odd or even.

For example, when the number of multiples of $(\mathrm{p})$ is even such as 6

$$
\text { p, 2p, 3p, 4p, 5p, 6p }
$$

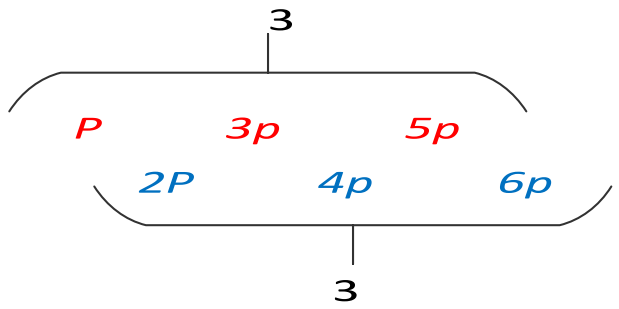

$3=3$

Figure 1

- If the number of multiples of (p) between (1) and (n) is odd, then the odd number of (p) multiples are more than its even number of multiples by one for whatever the state of (n), even or odd.

For example, when the number of multiples of (p) is odd such as 7 
1p, 2p, 3p, 4p, 5p, 6p, 7p

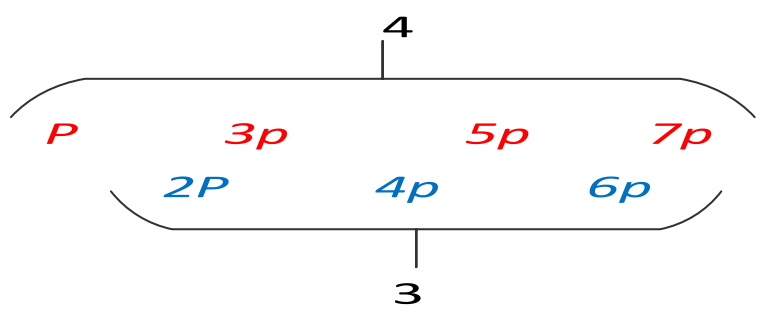

$4=3+1$

Figure 2

Step 2:Based on above $1 \& 2$ in step 1, number of multiples of (p) between (1) and (n) odd or even (depending on $n$ ) can be written as:

$\frac{\left\lfloor\frac{n}{p}\right\rfloor+s}{2}$

In which:

- When the result of $\left[\frac{n}{p}\right\rfloor$ is even, $\mathrm{S}=0$ for the dual cases of (n) due to the equalizing of multiples (odd and even) of (p).

$\frac{\left\lfloor\frac{n}{p}\right\rfloor}{2}$

- When the result of $\left[\frac{n}{p}\right]$ is odd,s $= \pm 1$ (the sign is referred to $n$ ) due to that odd multiples of (p) are more than even multiples of (p) by one.

If(n) is odd then $s=+1$

$\frac{\left\lfloor\frac{\mathrm{n}}{\mathrm{p}}\right\rfloor+1}{2}$

If(n) is even then $s=-1$

$\frac{\left\lfloor\frac{n}{p}\right\rfloor-1}{2}$

Step 3:Based on above a \& b in step 2, S can be written as:

$$
S=\frac{(-1)^{n+1}+(-1)^{\left\lfloor\frac{n}{p}\right\rfloor+n}}{2}
$$

Where this Value Will Achieve the Four Possibilities:

$$
\begin{aligned}
& \left\lfloor\frac{n}{p}\right\rfloor \text { Is even, (n) is even } \rightarrow s=0 \\
& \left\lfloor\frac{n}{p}\right\rfloor \text { Is even, (n) is odd } \rightarrow s=0 \\
& \left\lfloor\frac{n}{p}\right\rfloor \text { Is odd, (n) is even } \rightarrow s=-1
\end{aligned}
$$


$\left\lfloor\frac{n}{p}\right\rfloor$ Is odd, (n) is odd $\rightarrow s=+1$

Proof:

Assume: $h, f \in \mathbb{Z}$

And: $\left\lfloor\frac{n}{p}\right\rfloor=2 h$ if even, $\left\lfloor\frac{n}{p}\right\rfloor=2 h+1$ if odd.

And: $n=2 f$ if even, $: n=2 f+1$ if odd.

- If $\left\lfloor\frac{n}{p}\right\rfloor$ is even, then $s=0$, for the dual cases (even and odd) of (n).

a) When (n) is odd then :

$$
\begin{aligned}
S & =\frac{(-1)^{(2 f+1)+1}+(-1)^{2 h+(2 f+1)}}{2} \\
& =\frac{(-1)^{2 f+2}+(-1)^{2 h+2 f+1}}{2} \\
& =\frac{+1-1}{2} \\
& =0
\end{aligned}
$$

b) When (n) is even, then:

$$
\begin{aligned}
S & =\frac{(-1)^{2 f+1}+(-1)^{2 h+2 f}}{2} \\
& =\frac{-1+1}{2} \\
& =0
\end{aligned}
$$

- If $\left\lfloor\frac{n}{p}\right\rfloor$ is odd, then $s=\mp 1$, (Sign is referred to $\left.\mathrm{n}\right)$.

a) if (n) is odd, then $s=+1$

$$
\begin{aligned}
S & =\frac{(-1)^{(2 f+1)+1}+(-1)^{(2 h+1)+(2 f+1)}}{2} \\
& =\frac{(-1)^{2 f+2}+(-1)^{2 h+2 f+2}}{2} \\
& =\frac{+1+1}{2}=\frac{2}{2}=1
\end{aligned}
$$

b) if (n) is odd, then $s=-1$

$$
\begin{aligned}
S & =\frac{(-1)^{2 f+1}+(-1)^{(2 h+1)+2 f}}{2} \\
& =\frac{(-1)^{2 f+1}+(-1)^{2 h+2 f+1}}{2} \\
& =\frac{-1-1}{2}=\frac{-2}{2}=-1
\end{aligned}
$$


Step 4: By substituting of value (S),get multiples of $p$, and it the first term of the exponent of (p) where it is the prime number factor in (n!!) as:

$\frac{\left\lfloor\frac{n}{p}\right\rfloor+\frac{(-1)^{n+1}+(-1)^{\left\lfloor\frac{n}{p}\right\rfloor+n}}{2}}{2}$

Where all other terms of exponent of (p) are in the same manner.

Step 5: By summing multiples of $p$ with multiples of $p^{2}$ with multiples of $p^{3}$ and so on up to multiples of $p^{\left.\mid \log _{p} n\right\rfloor}($ the rationale for Legendre's formula), get the exponent of $\mathrm{p}$ the factor in $n !$ :

$v_{p}(n ! !)=\frac{\left\lfloor\frac{n}{p}\right\rfloor+\frac{(-1)^{n+1}+(-1)^{\left\lfloor\frac{n}{p}\right\rfloor+n}}{2}}{2}+\frac{\left\lfloor\frac{n}{p^{2}}\right\rfloor+\frac{(-1)^{n+1}+(-1)^{\left\lfloor\frac{n}{p^{2}}\right\rfloor+n}}{2}}{2}$

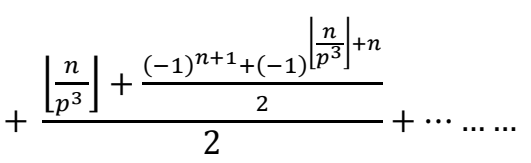

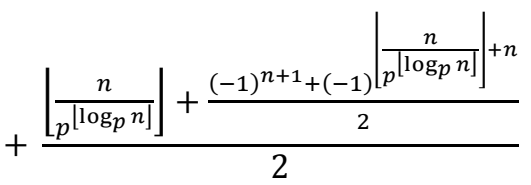

So:

$v_{p}(n ! !)=\sum_{k=1}^{\left\lfloor\log _{p} n\right\rfloor} \frac{\left\lfloor\frac{n}{p^{k}}\right\rfloor+\frac{(-1)^{n+1}+(-1)^{\left\lfloor\frac{n}{p^{k}}\right\rfloor+n}}{2}}{2}$

Example 1: if $n=100$, Then the prime numbers between (n) and (1)

is: $2,3,5,7,11,13,17,19,23,29,31,37,41,43,47,53,59,61,67,71,73,79,83,97$ The exponents of this prime numbers (Except for 2) can be found by applying the formula:

$$
v_{p}(n ! !)=\sum_{k=1}^{\left\lfloor\log _{p} n\right\rfloor} \frac{\left\lfloor\frac{n}{p^{k}}\right\rfloor+\frac{(-1)^{n+1}+(-1)}{2}\left\lfloor\frac{n}{p^{k}} \mid+n\right.}{2}
$$

As follows:

Table 1

\begin{tabular}{|c|c|c|c|}
\hline $\mathbf{P}$ & $v_{p}(100 ! !)$ & $\mathrm{p}$ & $v_{p}(100 ! !)$ \\
\hline 3 & 24 & 43 & 1 \\
\hline 5 & 12 & 47 & 1 \\
\hline 7 & 8 & 53 & 0 \\
\hline 11 & 4 & 59 & 0 \\
\hline 13 & 3 & 61 & 0 \\
\hline 17 & 2 & 67 & 0 \\
\hline 19 & 2 & 71 & 0 \\
\hline 23 & 2 & 73 & 0 \\
\hline 29 & 1 & 79 & 0 \\
\hline 31 & 1 & 83 & 0 \\
\hline
\end{tabular}




\begin{tabular}{|l|l|l|l|}
\hline 39 & 1 & 97 & 0 \\
\hline 41 & 1 & & \\
\hline
\end{tabular}

\subsection{Exception}

In the case of $p=2$, we apply the formula for Legendre, because number 2 is the a unique first prime number, so its multiples must be even, not like other prime numbers, whose multiples are odd and even alternately because they are odd.With the condition that (n) be even.

$v_{2}(n ! !)=\sum_{k=1}^{\left\lfloor\log _{2} n\right\rfloor}\left\lfloor\frac{n}{2^{k}}\right\rfloor ;$ is even number.

Example 2:if $n=100$ (100 is even number), then exponent of prime number 2 found by applying the Legendre's formula as follows:

$v_{2}(n ! !)=\sum_{k=1}^{\left\lfloor\log _{2} 100\right\rfloor}\left\lfloor\frac{100}{2^{k}}\right\rfloor=97$

\section{GENERALIZATION OF LEGENDER'S FORMULA TO THE MULTIFACTORIAL}

\subsection{Definition of MultiFactorial}

The multifactorial $n !^{q}$ is the product of $\mathrm{n}$ times the smaller numbers, where the differences between these numbers equal $\mathrm{q}$, which is the generalized concept of the factorial $\mathrm{n}$ ! When $q=1$, the multifactorial is factorial $n !^{1}=n ![2]$

$n !^{q}=\left\{\begin{aligned} & n, 0<n \leq q \\ & n\left((n-q) !^{q}\right), n>q\end{aligned}\right.$

It can be written as:

$n !^{q}=\prod_{m=0}^{\left\lceil\frac{n}{q}\right\rceil-1}(n-m q)=(n)(n-q)(n-2 q)(n-3 q) \ldots \ldots \ldots\left(n-\left(\left\lceil\frac{n}{q}\right\rceil-1\right) q\right)$

Suppose that the prime numbers between $\mathrm{n}$ and 1 are:

$p_{1}, p_{2}, p_{3}, p_{4}, p_{5}, p_{6}, p_{7}, \ldots \ldots, p_{m}$

Where m: number of primes between $\mathrm{n}$ and 1

Since the numbers between $\mathrm{n}$ and 1 are made up of prime numbers between $\mathrm{n}$ and 1 , the multifactorial $n !^{q}$ can be written as:

$$
p_{1}^{z_{1}} \times p_{2}^{z_{2}} \times p_{3}^{z_{3}} \times p_{4}^{z_{4}} \times p_{5}^{z_{5}} \times p_{6}^{z_{6}} \times p_{7}^{z_{7}} \times \ldots \ldots \ldots \times p_{m}^{z_{m}}
$$

\subsection{ProposedTheorem}

The exponent of the prime number $\mathrm{p}$ between $\mathrm{n}$ and 1 factor in the multifactorial $n !^{q}$ is given by the following relationship:

$v_{p}\left(n !^{q}\right)=\sum_{k=1}^{\left\lfloor\log _{p} n\right\rfloor} \sum_{m=0}^{\left\lfloor\frac{n}{q} \mid-1\right.}\left\lfloor\frac{\left\lfloor\frac{n-m q}{p^{k}}\right\rfloor}{\left(\frac{n-m q}{p^{k}}\right)}\right\rfloor$ 
Where:

$\mathrm{p}$ is prime number between $\mathrm{n}$ and 1

$\mathrm{n}$ is positive integer

$q$ the order of the multifactorial

$v_{p}(n !)$ Is the exponent of prime number $\mathrm{p}$ factor in $n !^{q}$

\section{Proof:}

The multifactorial $n !^{q}$ is equal:

$n !^{q}=\prod_{m=0}^{\left\lceil\frac{n}{q}\right\rceil-1}(n-m q)=(n)(n-q)(n-2 q)(n-3 q) \ldots \ldots \ldots\left(n-\left(\left\lceil\frac{n}{q}\right\rceil-1\right) q\right)$

So the factors of $n !^{q}$ are:

$(n),(n-q),(n-2 q),(n-3 q), \ldots \ldots \ldots,\left(n-\left(\left\lceil\frac{n}{q}\right\rceil-1\right) q\right)$

Step 1: Dividing the factors by the prime number $\mathrm{p}$ :

The multiplier factor of $\mathrm{p}$, divided by $\mathrm{p}$ produces an integer

$\frac{n-m q}{p} \in \mathbb{Z} ; \operatorname{if}(n-m q)$ is multiple of $p$

The non-multiplying factor of $\mathrm{p}$, divided by $\mathrm{p}$ produces an incorrect number

$\frac{n-m q}{p} \notin \mathbb{Z} ; \operatorname{if}(n-m q)$ is not multiple of $p$

Step 2: By dividing the nearest smaller integer (floor function [5]) of the product by the original

The new product is equal to one if the factor is a multiple of $p$

if $(n-m q)$ is multiple of $p$

$\Rightarrow \frac{n-m q}{p} \in \mathbb{Z}$

$\Rightarrow\left\lfloor\frac{n-m q}{p}\right\rfloor=\frac{n-m q}{p}$

$\Rightarrow \frac{\left\lfloor\frac{n-m q}{p}\right\rfloor}{\left(\frac{n-m q}{p}\right)}=1$

The new product is less than one if the factor is non-multiple of $p$

$\operatorname{if}(n-m q)$ is not multiple of $p$

$\Rightarrow \frac{n-m q}{p} \notin \mathbb{Z}$ 
$\Rightarrow\left\lfloor\frac{n-m q}{p}\right\rfloor<\frac{n-m q}{p}$

$\Rightarrow \frac{\left\lfloor\frac{n-m q}{p}\right\rfloor}{\left(\frac{n-m q}{p}\right)}<1$

Step 3: By taking the nearest integer it results (floor function [5]):

The product remains equal to one if the factor is a multiple of $\mathrm{p}$

if $(n-m q)$ is multiple of $p$

$\Rightarrow \frac{n-m q}{p} \in \mathbb{Z}$

$\Rightarrow\left\lfloor\frac{n-m q}{p}\right\rfloor=\frac{n-m q}{p}$

$\Rightarrow \frac{\left\lfloor\frac{n-m q}{p}\right\rfloor}{\frac{n-m q}{p}}=1$

$\Rightarrow\left\lfloor\frac{\left\lfloor\frac{n-m q}{p}\right\rfloor}{\frac{n-m q}{p}}\right\rfloor=1$

If the factor is not a multiple of $p$ then the result is zero

$\operatorname{if}(n-m q)$ is not multiple of $p$

$\Rightarrow \frac{n-m q}{p} \notin \mathbb{Z}$

$\Rightarrow\left\lfloor\frac{n-m q}{p}\right\rfloor<\frac{n-m q}{p}$

$\Rightarrow \frac{\left\lfloor\frac{n-m q}{p}\right\rfloor}{\left(\frac{n-m q}{p}\right)}<1$

$\Rightarrow\left\lfloor\frac{\left\lfloor\frac{n-m q}{p}\right\rfloor}{\frac{n-m q}{p}}\right\rfloor=0$

Step 4: By adding the products of all the intuitive factors of $n !^{q}$ produces the number of multiples of $\mathrm{p}$ within factors of $n !^{q}$

$a=\left\lfloor\frac{\left\lfloor\frac{n}{p}\right\rfloor}{\left(\frac{n}{p}\right)}\right\rfloor+\left\lfloor\frac{\left\lfloor\frac{n-q}{p}\right\rfloor}{\left(\frac{n-q}{p}\right)}\right\rfloor+\left\lfloor\frac{\left\lfloor\frac{n-2 q}{p}\right\rfloor}{\left(\frac{n-2 q}{p}\right)}\right\rfloor+\cdots+\left\lfloor\frac{\left\lfloor\frac{n-\left(\left\lfloor\frac{n}{q} \mid-1\right) q\right.}{p}\right\rfloor}{\left(\frac{n-\left(\left\lfloor\frac{n}{q} \mid-1\right) q\right.}{p}\right)}\right\rfloor$ 
$=\sum_{m=0}^{\left\lceil\frac{n}{q}\right\rceil-1}\left\lfloor\frac{\left\lfloor\frac{n-m q}{p}\right\rfloor}{\left(\frac{n-m q}{p}\right)}\right\rfloor$

Where: $a$ is the number of $\mathrm{p}$ multiples within factors ofn! ${ }^{\mathrm{q}}$.

Similar case if the exponent increases in $\mathrm{p}$

$b=\left\lfloor\frac{\left.\frac{n}{p^{k}}\right\rfloor}{\left(\frac{n}{p^{k}}\right)}\right\rfloor+\left\lfloor\frac{\left.\frac{n-q}{p^{k}}\right\rfloor}{\left(\frac{n-q}{p^{k}}\right)}\right\rfloor+\left\lfloor\frac{\left\lfloor\frac{n-2 q}{p^{k}}\right\rfloor}{\left(\frac{n-2 q}{p^{k}}\right)}\right\rfloor+\cdots+\left\lfloor\frac{\left\lfloor\frac{n-\left(\frac{n}{q} \mid-1\right) q}{p^{k}}\right\rfloor}{\left(\frac{n-\left(\frac{n}{q} \mid-1\right) q}{p^{k}}\right)}\right\rfloor$

$=\sum_{m=0}^{\left\lceil\frac{n}{q}\right\rceil-1}\left\lfloor\frac{\left\lfloor\frac{n-m q}{p^{k}}\right]}{\left(\frac{n-m q}{p^{k}}\right)}\right\rfloor$

Where: $\mathrm{b}$ is number of multiples of $p^{k}$ within factors of $n !^{q}$.

Step 5: By summing multiples of $p$ with multiples of $p^{2}$ with multiples of $p^{3}$ and so on up to multiples of $p^{\left.\mid \log _{p} n\right\rfloor}($ the rationale for Legendre's formula), get the exponent of $\mathrm{p}$ the factor in $n !^{q}$ :

$v_{p}\left(n !^{q}\right)=\sum_{m=0}^{\left\lceil\frac{n}{q}\right\rceil-1}\left\lfloor\frac{\left[\frac{n-m q}{p}\right\rfloor}{\left(\frac{n-m q}{p}\right)}\right\rfloor+\sum_{m=0}^{\left\lceil\frac{n}{q}\right\rceil-1}\left\lfloor\frac{\left\lfloor\frac{n-m q}{p^{2}}\right]}{\left(\frac{n-m q}{p^{2}}\right)}\right\rfloor+\sum_{m=0}^{\left\lceil\frac{n}{q}\right\rceil-1}\left\lfloor\left\lfloor\frac{\left.\frac{n-m q}{p^{3}}\right\rfloor}{\left(\frac{n-m q}{p^{3}}\right)}\right\rfloor+\cdots\right.$

$+\sum_{m=0}^{\left\lceil\frac{n}{q} \mid-1\right.}\left\lfloor\frac{\left\lfloor\frac{n-m q}{\left.p^{\mid \log p n}\right\rfloor}\right\rfloor}{\left(\frac{n-m q}{p^{\mid \log p n}}\right)}\right\rfloor$

So:

$v_{p}\left(n !^{q}\right)=\sum_{k=1}^{\left\lfloor\log _{p} n\right\rfloor} \sum_{m=0}^{\left\lfloor\frac{n}{q}\right\rfloor-1}\left\lfloor\frac{\left\lfloor\frac{n-m q}{p^{k}}\right\rfloor}{\left(\frac{n-m q}{p^{k}}\right)}\right\rfloor$

\section{Example 3:}

if $n=150$, and $q=7$, find the exponents of the prime numbers between $\mathrm{n}$ and 1 factor in $\mathrm{n} !^{\mathrm{q}}$

Solution:

The prime numbers between $\mathrm{n}$ and 1 are:2, 3, 5, 7, 11, 13, 17, 19, 23, 29,31, 37, 41, 43, 47, 53, 59, 61, 67, 71, 73, 79, 83, 89, 97, $101,103,107,109,113,127,131,137,139,149$

The exponents of these prime numbers factor in $150 !^{7}$ can be found by applying the formula:

$v_{p}\left(n !^{q}\right)=\sum_{k=1}^{\left\lfloor\log _{p} n\right\rfloor} \sum_{m=0}^{\left\lfloor\frac{n}{q}\right\rceil-1}\left\lfloor\frac{\left\lfloor\frac{n-m q}{p^{k}}\right\rfloor}{\left(\frac{n-m q}{p^{k}}\right)}\right\rfloor$ 
As follows:

Table 2

\begin{tabular}{|c|c|c|c|}
\hline $\mathbf{P}$ & $\boldsymbol{v}_{\boldsymbol{p}}\left(\mathbf{1 5 0} !^{\mathbf{7}}\right)$ & $\mathbf{p}$ & $\boldsymbol{v}_{\boldsymbol{p}}\left(\mathbf{1 5 0} !^{\mathbf{7}}\right)$ \\
\hline 2 & 20 & 67 & 0 \\
\hline 3 & 11 & 71 & 0 \\
\hline 5 & 6 & 73 & 1 \\
\hline 7 & 0 & 79 & 0 \\
\hline 11 & 2 & 83 & 0 \\
\hline 13 & 2 & 89 & 0 \\
\hline 17 & 2 & 97 & 0 \\
\hline 19 & 1 & 101 & 1 \\
\hline 23 & 1 & 103 & 0 \\
\hline 29 & 1 & 107 & 0 \\
\hline 31 & 1 & 109 & 0 \\
\hline 37 & 0 & 113 & 0 \\
\hline 41 & 0 & 127 & 0 \\
\hline 43 & 1 & 131 & 0 \\
\hline 47 & 1 & 137 & 0 \\
\hline 53 & 0 & 139 & 0 \\
\hline 59 & 1 & 149 & 0 \\
\hline 61 & 1 & & \\
\hline
\end{tabular}

\section{CONCLUSIONS}

The Legendre's Formula is extended by formulating new relationshipfor finding the exponents of prime numbers of double factorial. The proposed theorem is proofed and validated throughout numerical example.

The formula as follows:

$$
v_{p}(n ! !)=\sum_{k=1}^{\left\lfloor\log _{p} n\right\rfloor} \frac{\left\lfloor\frac{n}{p^{k}}\right\rfloor+\frac{(-1)^{n+1}+(-1)^{\left\lfloor\frac{n}{p^{k}}\right\rfloor+n}}{2}}{2}
$$

A generalization of the Legendre's Formula has been introduced by expressing a new formula for the exponents of prime numbers of multifactorial. The new formulahas been proved and validated throughout numerical example.

The formula as follows:

\section{REFERENCES}

$$
v_{p}\left(n !^{q}\right)=\sum_{k=1}^{\left\lfloor\log _{p} n\right\rfloor} \sum_{m=0}^{\left\lfloor\frac{n}{q}\right\rceil-1}\left\lfloor\frac{\left\lfloor\frac{n-m q}{p^{k}}\right\rfloor}{\left(\frac{n-m q}{p^{k}}\right)}\right\rfloor
$$

1. Ronald L. Graham, Donald E. Knuth, and O. Patashnik, C O N C R E T E M A THE M A T I C S, 2nd ed. New York: ADDISON-WESLEY PUBLISHING COMPANY, 1994.

2. E. W. Weisstein. (2001). Factorial. Available: https://mathworld.wolfram.com/Factorial.html.

3. Kornilowicz and P. Rudnicki, "Fundamental Theorem of Arithmetic," Formalized Mathematics, vol. 12, pp. 179186, 2004.

4. L. E. Dickson, History of the Theory of Numbers vol. 1: Carnegie Institution of Washington, 1919.

5. Weisstein and E. W. (2004). floor function. Available: https://mathworld.wolfram.com/FloorFunction.html.

6. L. Tesler.(1961). Factorials and Primes. Available: http://www.nomodes.com/Larry_Tesler_Personal/Math_Bulletin.html. 
7. D. CALLAN. (2009, 30 April 2020). A Combinatorial Survey of Identities for the Double Factorial. Available: https://arxiv.org/abs/0906.1317v1,Cornell University.

8. E. W. Weisstein. (2002). Double Factorial. Available: https://mathworld.wolfram.com/DoubleFactorial.html. 